\title{
El papel de los padres y los amigos en el consumo temprano de alcohol
}

\author{
SíGRID GALLEGo MOYA \\ moyam@uji.es \\ MANUEL IGNACIO IBÁÑEZ RIBES \\ iribes@uji.es
}

\section{Resumen}

Introducción: El consumo de alcohol en la adolescencia es atribuible a la influencia de variables de tipo social, sobre todo del grupo de iguales y del ámbito familiar. Específicamente, el consumo de los amigos resulta ser la variable más importante en las primeras etapas del consumo de alcohol. No obstante, el uso que hacen los padres de esta sustancia y el empleo de estilos permisivos e inconsistentes en la educación de sus hijos, parecen favorecer también el inicio temprano al consumo de alcohol. Por tanto, el objetivo del presente trabajo es estudiar la influencia de los estilos educativos y el consumo de alcohol paterno, así como del consumo de los amigos, en las primeras etapas del uso de alcohol durante la adolescencia. Método: 330 adolescentes jóvenes ( $M=13,05 ; 57,27 \%$ chicas) completaron diferentes cuestionarios que evaluaban los estilos educativos parentales percibidos (EMBU y APQ), así como su consumo de alcohol, el de sus amigos y el de sus padres (AIS-UJI). Resultados: El modelo de ecuación estructural propuesto mostró como el consumo de los iguales se asociaba directamente y de forma muy importante al consumo de alcohol de los jóvenes. Además, existía una relación indirecta entre consumo de los padres y de los amigos, que vendría mediada por los estilos educativos permisivos. Conclusiones: El presente estudio revela que los padres más consumidores tienden a mostrar estilos educativos más permisivos e inconsistentes, lo que favorecería la afiliación con amigos más bebedores y un consumo de alcohol más temprano. Estos resultados resultan de especial importancia para el desarrollo de estrategias de prevención más personalizadas y eficaces en el consumo de sustancias de jóvenes adolescentes.

Palabras clave: alcohol, estilos educativos, padres, adolescentes, iguales.

\section{Abstract}

Introduction: Alcohol consumption in adolescence is influenced by social variables, such as peer group and family. Thus, friends' alcohol use is the most important factor in the early stages of alcohol consumption. Moreover, parents' drinking behaviour and permissive and inconsistent rearing style also seem to encourage the early onset of alcohol consumption. The aim of this research work was to study the influence of parenting style, and parents' and friends' alcohol use, on adolescent drinking behaviour, that is to say in the early stages of alcohol use. Method: 330 young adolescents 
(Mage $=13.05$ years; $57.27 \%$ females) completed two perceived parenting style questionnaires (EMBU and $\mathrm{APQ}$ ), and a scale assessing own, friends' and parents' alcohol use (AIS-UJI). Results: The proposed structural equation model showed that peers' consumption was associated directly and very significantly with adolescents' alcohol use. In addition, there was an indirect relationship between parents' and friends' consumption, which was mediated by permissive parenting style. Conclusions: The present study reveals that those parents that consume more alcohol tend to use permissive and inconsistent parenting style, favouring their offspring's affiliation with drinking friends and an early onset of alcohol use. These results are particularly important for developing more personalised and successful prevention programmes for alcohol misuse in young adolescents.

Keywords: alcohol, educational styles, parents, adolescence, peers.

\section{Introducción}

El alcohol es la sustancia más consumida en nuestro país durante la adolescencia. Las encuestas nacionales muestran que la edad con la que los jóvenes se inician al consumo de alcohol es más temprana que para el resto de sustancias psicoactivas, 13,9 años aprox., y que a los 15 años de edad ya mantienen un patrón de consumo semanal (Plan Nacional sobre Drogas, 2012).

Este inicio temprano y el mantenimiento de su uso en jóvenes no solo tienen importantes repercusiones en el momento de su uso, sino que constituye un factor de riesgo para futuros problemas (p.e. el consumo de sustancias ilícitas y/o comportamientos peligrosos para la salud). Por ello, y con el fin de prevenir este tipo de problemática futura, resulta de suma importancia identificar las variables que intervienen en este tipo de comportamientos.

Los estudios de genética cuantitativa permiten cuantificar, aproximadamente, el grado de influencia que los factores genéticos y ambientales tienen en los diferentes patrones de consumo alcohólico desde una perspectiva de diferencias individuales. Así, este tipo de estudios indicaría que los factores más relevantes al inicio del consumo de alcohol durante edades tempranas, serían aquellos de carácter más psicológico y social, mientras que a edades más adultas o en patrones de consumo de alcohol ya establecidos, los factores genéticos cobrarían mayor relevancia; mientras que los factores ambientales, aun siendo importantes, tendrían un menos peso que en etapas anteriores (Kendler, Schmitt, Aggen, y Prescott, 2008).

Como vemos en la figura 1 , los factores más relevantes en el inicio temprano del consumo de alcohol, a los 14-15 años, los factores que mayor porcentaje de varianza explicarían de estas conductas serían los aspectos sociales o ambientales. Mientras que a edades más adultas o con patrones de consumo ya establecidos, serían los factores genéticos aquellos que cobrarían mayor relevancia y, en cambio, el ambiente, aun siendo importante, tendría un peso menor que a edades tempranas. En definitiva, el inicio al consumo de alcohol a edades tempranas estaría influido por factores ambientales, tanto familiar como extra-familiar. No obstante, cuando los jóvenes transitan del uso experimental y esporádico del alcohol a un consumo mucho más estable, la influencia genética de estas conductas se incrementa y la influencia del ambiente familiar comienza a decrecer, siendo el ambiente no compartido el que toma una mayor relevancia (Kendler y cols., 2008; Rose y Dick, 2004). 


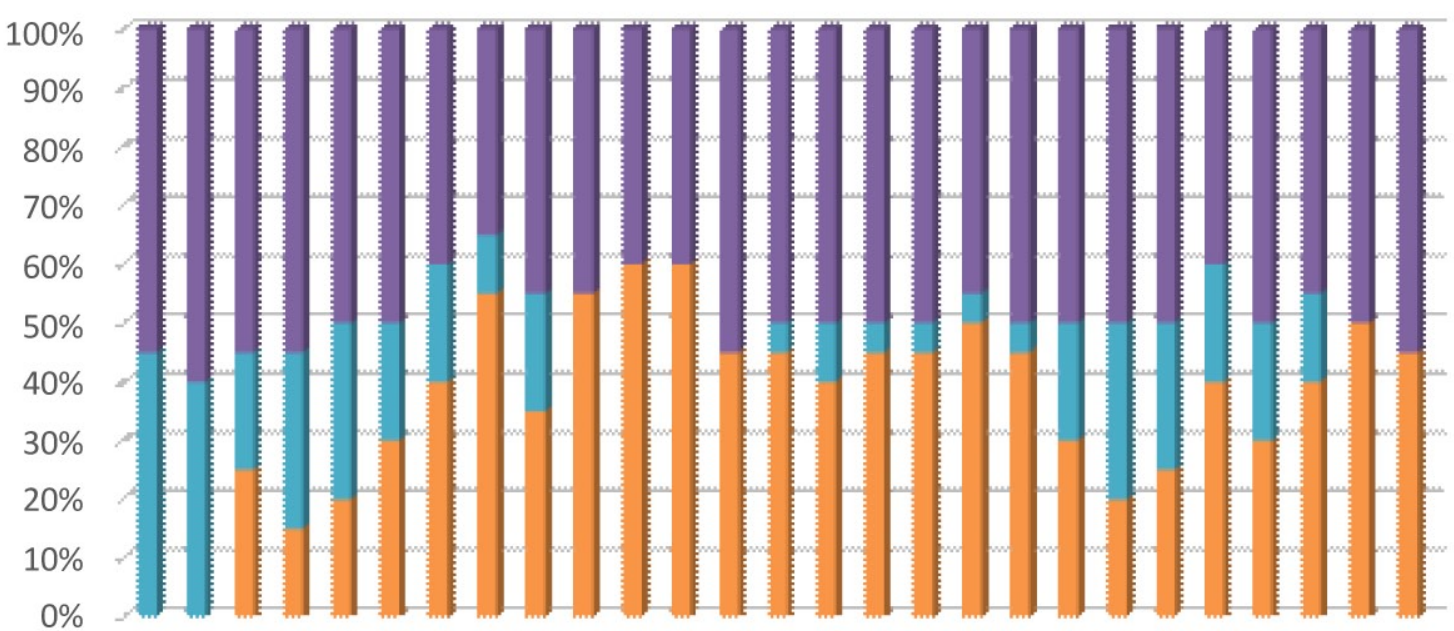

141516171819202122232425262728293031323334353637383940

- Heredabilidad Ambiente compartido Ambiente no compartido

Figura 1. Porcentaje de varianza del consumo de alcohol en diferentes grupos de edad que se explica por factores genéticos y ambientales. Adaptada de Kendler y cols. (2008)

De entre diferentes variables de carácter social, encontramos el consumo de alcohol paterno. Como ejemplo, en un estudio sobre la relación entre el consumo de alcohol de padres e hijos, y con una muestra representativa de EEUU de más de 10.000 parejas, mostraron que los adolescentes que más alcohol consumieron eran hijos de padres que parecían mostrar sintomatología de dependencia alcohólica (National Survey on Drug Use and Health, 2013).

Otra variable familiar ampliamente estudiada son los estilos educativos paternos. Por ejemplo, un estudio con muestra española que relaciona distintos estilos de crianza con el consumo de alcohol indicaba que la baja monitorización o la permisividad hacia los hijos se relacionan de forma directa y significativa con el consumo de alcohol en jóvenes (Becoña y cols., 2013).

Por otra parte, y dentro del ambiente extra-familiar, el consumo de alcohol de los amigos es probablemente la variable más fuertemente asociada al consumo de los jóvenes, tanto al inicio como en el mantenimiento de esta conducta (Borsari y Carey, 2001). Estudios transversales indican que existe correlación en esta conducta desde la adolescencia hasta el inicio de la adultez, desde los 12 a 20 años aprox. (Ibáñez y cols., 2008), y distintos estudios longitudinales muestran cómo el consumo de los amigos influye en el consumo propio, y viceversa (Simons-Morton y Chen, 2006; Wills y Cleary, 1999).

Por tanto, el objetivo de este estudio fue analizar la influencia de las variables del entorno familiar (consumo de alcohol de los padres y estilos de crianza) y del entorno social (consumo de alcohol de los amigos) en el consumo de alcohol de los jóvenes. Nuestra hipótesis fue que el consumo de los padres predeciría un estilo educativo más permisivo, lo que, a su vez, facilitaría que los hijos se afiliaran con amigos más bebedores. Esta sería la variable que, de forma más fuerte y directa, se asociaría con el consumo de alcohol propio. 


\section{Método}

\section{Muestra y procedimiento}

La muestra estaba compuesta de 330 estudiantes de Enseñanza Secundaria Obligatoria de centros de la Comunidad Valenciana (España), tanto de zonas urbanas como rurales. El $57,80 \%$ eran chicas y la media de edad era 13,05 años $(d$. t. $=0,56)$. Los cuestionarios se administraron de forma colectiva, motivando a los participantes y asegurándoles la confidencialidad de los resultados.

\section{Instrumentos}

- El AIS-UJI (Ortet y cols., 2002) evalúa el consumo de alcohol de los padres, los iguales y los adolescentes con periodicidad semanal (entre semana y fin de semana). Las Unidades de Bebida Estándar (UBE; Rodríguez-Martos, Gual y Llopis, 1999) se calcularon a partir de una pregunta semiestructurada en la que los adolescentes informaban el número de vasos de vino, cervezas, combinados y licores sin combinar que tomaban durante el fin de semana. El vino y la cerveza se contabilizan como una UBE, mientras el combinado y los licores sin combinar ('chupitos') se contabilizaban como dos UBE (Rodríguez-Martos, Gual y Llopis, 1999). En España una UBE equivale a 10 gramos de alcohol (Llopis, Gual y Rodríguez-Martos, 2000).

- El EMBU-SF (Arrindell y cols., 1999) y APQ (Shelton, Frick y Whooton, 1996). El EMBU-SF evalúa tres estilos educativos diferentes: calidez emocional, sobreprotección y rechazo, tanto del padre como de la madre. Por su parte, el APQ evalúa el castigo, la inconsistencia disciplinaria, el estilo educativo positivo de ambos padres conjuntamente y la implicación de la madre y el padre de forma separado.

\section{Análisis}

En primer lugar, se realizó un análisis factorial exploratorio (EFA) en el que se incluyeron las doce escalas de estilos educativos evaluadas con ambos instrumentos. Se utilizó el método de extracción de ejes principales y rotación oblimin, y para la extracción del número de factores se realizó un análisis paralelo con el software Monte Carlo PCA for Parallel Analysis, versión 2.5.

A continuación, se calcularon los datos descriptivos y las correlaciones bivariadas de las variables de consumo de los padres, los amigos y los jóvenes, y los factores resultantes del EFA de estilos educativos parentales, con el paquete estadístico IBM SPSS statistics versión 21 (IBM, 2012).

Además, para analizar los efectos directos y la posible existencia de un efecto de moderación o interacción entre las variables, se hipotetizó un modelo de ecuaciones estructurales con el EQS 6.1 (EQS; Bentler y Wu, 2003). Debido a que los análisis exploratorio de los datos no indicaron no normalidad en la distribución de los datos (coeficientes de Mardia>40; Mardia, 1970), la estimación de las relaciones se llevó a cabo mediante los métodos robustos de Satorra-Bentler (Satorra y Bentler, 2001).

Todas las puntuaciones de las variables utilizadas para los análisis de este estudio fueron centradas por la media, se les restó el valor medio de la variable, de forma que las puntuaciones más altas equivalen a más del constructo medido (Cooper, 2000). 


\section{Resultados}

\section{Análisis factorial exploratorio (EFA)}

Este análisis indica que la agrupación de las variables de los cuestionarios EMBU-SF y APQ está formada por 4 factores cuyos autovalores son mayores a la unidad y saturan por encima de 0,46. El conjunto explica el 76,62 \% de la varianza total acumulada (factor $1=33,07$ $\%$; factor2 $=22,40 \%$; factor3 $=6,42 \%$; factor4 $=5,26 \%$ ).

La significación del estadístico $X^{2}\left(X^{2}=25424,60 ;\right.$ g.l.: $\left.66 ; p=0,00\right)$ y el índice $\operatorname{KMO}(0,68)$ indicaron que el método de extracción que utilizamos, oblimin directo, era adecuado. Esta estructura de 4 factores fue confirmada a través del gráfico de sedimentación un análisis paralelo.

En conclusión, el EFA mostró la adecuación de agrupar las escalas en cuatro factores de estilos educativos a los que se etiquetó como: «calidez» (implicación, calidez, estilo positivo), «sobreprotección», «permisividad» (escasa supervisión, inconsistencia disciplinaria) y «castigo» (rechazo, castigo).

\section{Análisis descriptivos}

La media de consumo de alcohol de los participantes en una semana fue de 1,83 UBE (unidades bebida estándar) (d. t. = 5,32) (Rodríguez-Martos y cols., 1999). Del total de la muestra, 257 estudiantes informaron que no bebían alcohol $(77,88 \%)$.

El 64,24 \% de los jóvenes evaluados informaron que no tenían ningún amigo que consumiera alcohol y el $13,33 \%$ indicaron que sus padres no consumían nada de alcohol semanalmente.

Tabla 1

Medias y desviaciones típicas de toda la muestra en cada variable, así como medias y desviaciones típicas por género, pruebas $\mathrm{t} y \mathrm{~d}$ de Cohen

\begin{tabular}{lccccc}
\hline & $\begin{array}{c}\text { Toda la muestra } \\
(\mathrm{N}=330)\end{array}$ & Chicos $(\mathrm{N}=141)$ & Chicas $(\mathrm{N}=189)$ & $t$ & $d$ \\
\hline $\begin{array}{l}\text { Consumo alcohol } \\
\text { madre (CAM) }\end{array}$ & $2,61(2,26)$ & $1,28(1,61)$ & $1,11(1,51)$ & $2,73^{* *}$ & 0,11 \\
\hline $\begin{array}{l}\text { Consumo alcohol } \\
\text { padre (CAP) }\end{array}$ & $1,18(1,55)$ & $3,00(2,36)$ & $2,33(2,14)$ & 0,96 & 0,30 \\
\hline $\begin{array}{l}\text { Consumo alcohol } \\
\text { amigos (CAA) }\end{array}$ & $2,46(4,73)$ & $2,47(4,48)$ & $2,45(4,93)$ & 0,05 & 0,00 \\
\hline $\begin{array}{l}\text { Consumo alcohol } \\
\text { jóvenes (CAJ) }\end{array}$ & $1,83(5,32)$ & $2,56(6,93)$ & $1,30(3,63)$ & $2,14^{*}$ & 0,23 \\
\hline
\end{tabular}

Nota: ${ }^{*} p<, 05,{ }^{* *} p<, 01,{ }^{* * *} p<, 001$. Valores $d$ de Cohen de $, 20,, 50$ y, 80 , se corresponden con un tamaño del efecto pequeño, medio y grande, respectivamente (Cohen, 1992). 
Tabla2

Correlaciones bivariadas de Pearson

\begin{tabular}{lccccccc}
\hline & 2. & 3. & 4. & 5. & 6. & 7. & 8. \\
\hline 1. CAP & $0,526^{* *}$ & $0,195^{* *}$ & $0,174^{* *}$ & $-0,075$ & $-0,003$ & $0,283^{* *}$ & $0,115^{*}$ \\
\hline 2. CAM & - & 0,104 & $0,155^{* *}$ & $-0,046$ & 0,094 & $0,259^{* *}$ & $0,206^{* *}$ \\
\hline 3. CAA & & - & $0,615^{* *}$ & $-0,100$ & 0,039 & $0,305^{* *}$ & $0,112^{*}$ \\
\hline 4. CAJ & & & - & $-0,185^{* *}$ & 0,103 & 0,328 & $0,145^{* *}$ \\
\hline 5. Calidez & & & & - & $-0,080$ & $-0,304^{* *}$ & $-0,312^{* *}$ \\
\hline 6. Sobrepotección & & & & - & $0,234^{* *}$ & $0,483^{* *}$ \\
\hline 7. Permisividad & & & & & - & $0,448^{* *}$ \\
\hline 8. Castigo & & & & & & & \\
\hline
\end{tabular}

Nota: ${ }^{*} p<0,05,{ }^{* *} p<0,01,{ }^{* * *} p<0,001$

\section{Modelo de ecuaciones estructurales}

El análisis de ecuaciones estructurales mostró que el modelo hipotetizado se ajustaba de forma adecuada a nuestros datos (ver figura 2). El consumo de la madre se relacionó de manera directa con la sobreprotección, el castigo y con la permisividad, en este último también se relacionó el consumo del padre. Por su parte, la permisividad se relacionó con el consumo del grupo de amigos, y el consumo de los amigos se relacionó con el consumo de los jóvenes.

El estudio de los efectos indirectos mostró que la permisividad se relacionaba de forma indirecta con el consumo de alcohol de los jóvenes a través de la afiliación con iguales consumidores $(\beta=0,19 ; p<0,05)$. No obstante, el consumo de alcohol del padre, se relacionó de forma indirecta y parcial con el consumo de alcohol a través de la permisividad $(\beta=0.05$; $p<0,05)$ y el consumo del grupo de amigos $(\beta=0,07 ; p<0,05)$.

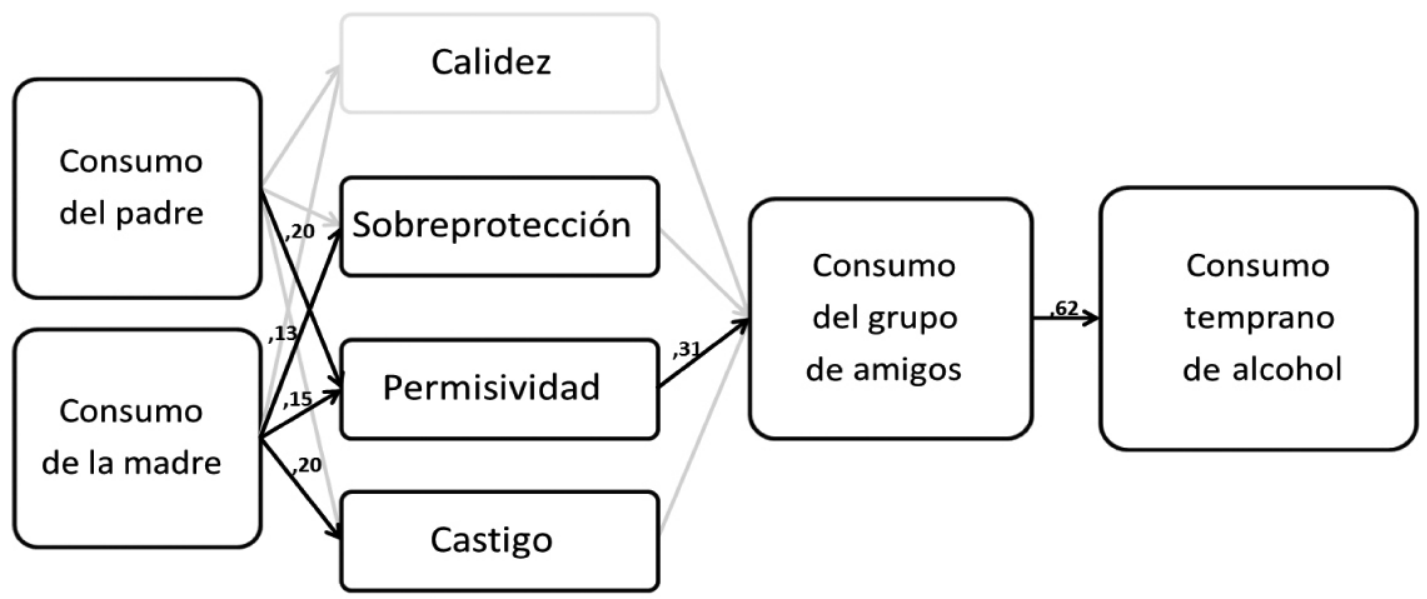

\begin{tabular}{|c|c|c|c|c|c|c|c|}
\hline $\mathrm{X}^{2}$ & $\mathrm{gl}$ & $\mathrm{P}$ & $\mathrm{X}^{2} / \mathrm{gl}$ & $\mathrm{CFI}$ & $\mathrm{IFI}$ & $\mathrm{NFI}$ & RMSEA \\
\hline 10,29 & 8 & 0,25 & 1,73 & 0,99 & 0,99 & 0,96 & $\begin{array}{c}0,03 \\
(0,00-0,08)\end{array}$ \\
\hline
\end{tabular}

Nota: sobre las líneas coeficientes $\beta$ estandarizados a ${ }^{*} p<0,05,{ }^{* *} p<0,01,{ }^{* * *} p<0,001$. Las correlaciones no significativas no se incluyen en la figura para facilitar su interpretación.

Figura 2. Modelo de ecuaciones estructurales 


\section{Discusión y conclusiones}

El objetivo del presente trabajo fue estudiar que variables ambientales, tanto familiares como extrafamiliares, son relevantes en el consumo temprano de alcohol en jóvenes. En concreto, se investigó el papel del consumo del grupo de iguales, así como el consumo de los padres y los estilos educativos que empleaban para criar a sus hijos.

En primer lugar, encontramos que el consumo de alcohol de los amigos explicaba casi el $11,8 \%$ de varianza del consumo propio, por lo que constituye la variable de riesgo más relevante en el consumo de alcohol temprano en jóvenes a través de una vía explicativa directa, de acuerdo con la literatura previa (Borsari y Carey, 2001).

Además, otras variables como el consumo de alcohol de los padres y los estilos educativos de carácter permisivo y de baja supervisión también mostraron ser relevantes, en línea con otros trabajos (Becoña y cols., 2013; National Survey on Drug Use and Health, 2013). No obstante, y algo que aporta el presente trabajo, es que esta influencia es de carácter indirecto, ya que estaría mediada por la afiliación con los iguales. Así, el consumo de los padres se asociaba con un estilo educativo más permisivo, el cual favorecía que los hijos se afiliaran con grupos de iguales que mostraban un mayor consumo de alcohol.

De forma más específica, encontramos que los padres que más bebían mostraban un estilo educativo más permisivo y de menos supervisión, al igual que la madre. Además, las madres más bebedoras también mostraban un estilo más castigador y más sobreprotector, aunque este tipo de crianza no influía, ni indirecta ni directamente, en el consumo de alcohol de los hijos. Dado que nuestro estudio es de tipo transversal, desconocemos si el hecho de consumir alcohol provocaría que los padres mostraran una mayor desinhibición conductual que se reflejaría en estilos más permisivos y castigadores, o que los padres muestran un mayor consumo y estilos educativos más negligentes debido a características de personalidad asociadas con ambos aspectos, como la baja amabilidad y baja responsabilidad (Huver, Otten, de Vries, y Engels, 2010; Malouff, Thorsteinsson, Schutte, Bhullar, y Rooke, 2010).

En definitiva, hemos encontrado un efecto directo y muy importante del grupo de amigos en el consumo temprano de alcohol en adolescentes. Además, los estilos educativos de carácter más permisivo mediarían en la relación entre el consumo de alcohol de los padres y la facilitación de la afiliación con otros jóvenes también consumidores de alcohol.

Estos resultados sugieren que en el diseño de estrategias de prevención e intervención eficaces deberían tener en cuenta no solo el consumo de padres y amigos, sino también los estilos educativos excesivamente permisivos, de baja monitorización y poca implicación y supervisión. Por ejemplo, estrategias que conjuntamente orienten su estrategia de prevención y tratamiento tanto a padres como a grupo de iguales serán más eficaces. Estas estrategias deberían contemplar aspectos de formación para los padres, no solo orientadas a una reducción del consumo en presencia de los hijos, sino en el entrenamiento de estrategias de crianza de mayor implicación emocional y control y supervisión, así como la inclusión de actividades de ocio alternativas al consumo de alcohol en el grupo de amigos.

\section{Referencias bibliográficas}

Arrindell, W. A., Sanavio, E., Aguilar, G., Sica, C., Hatzichristou, C.,... Van der Ende, J. (1999). The development of a short form of the EMBU: Its appraisal with students in Greece, Guatemala, Hungary and Italy. Personality and Individual Differences, 27, 613-628. 
Becoña, E., Martínez, Ú., Calafat, A., Fernández-Hermida, J. R., Juan, M., Sumnall, H., ... Gabrhelík, R. (2013). Parental permissiveness, control, and affect and drug use among adolescents. Psicothema, 25, 292-298. doi:10.7334/psicothema2012.294.

Bentler P. M. (2006). EQS 6 Structural Equations Program Manual. Encino, Multivariate Software Inc.

Bentler, P. M. y Wu, E. J. C. (2003). EQS structural equations program version 6.1. [Computer software]. Encino, CA: Multivariate Software.

Borsari, B., y Carey, K. B. (2001). Peer influences on college drinking: A review of the research. Journal of Substance Abuse, 13, 391-424.

Cohen, J. (1992). A power primer. Psychological Bulletin, 112, 155-159.

Cooper, M. L., Agocha, V. B. y Sheldon, M. S. (2000). A motivational perspective on risky behaviors: the role of personality and affect regulatory processes. Journal of Personality, 68, 1059-1088.

Huver, R. M. E., Otten, R., de Vries, H., y Engels, R. C. M. E. (2010). Personality and parenting style in parents of adolescents. Journal of Adolescence, 33, 395-402. doi:10.1016/j. adolescence.2009.07.012.

Ibáñez, M. I., Ruipérez, M. A., Villa, H., Moya, J. y Ortet, G. (2008). Personality and alcohol use. En G. J. Boyle, G. Matthews y D. H. Saklofske (eds.), Handbook of Personality Theory and Testing. Nueva York: Sage.

IBM Corp. Released (2012). IBM SPSS Statistics for Windows, version 21.0. Armonk, Nueva York: IBM Corp.

Kendler, K. S., Schmitt, E., Aggen, S. H., y Prescott, C. A. (2008). Genetic and Environmental Influences on Alcohol, Caffeine, Cannabis, and Nicotine Use from Early Adolescence to Middle Adulthood. Archives of General Psychiatry, 65, 674-682. doi:10.1001/archpsyc.65.6.674. Genetic.

Llopis Llàcer, J. J., Gual Solé, A. y Rodríguez-Martos Dauer, A. (2000). Registro del consumo de bebidas alcohólicas mediante la unidad de bebida estándar. Diferencias geográficas. Adicciones, 12, 11-19.

Malouff, J. M., Thorsteinsson, E. B., Schutte, N. S., Bhullar, N., y Rooke, S. E. (2010). The Five-Factor Model of personality and relationship satisfaction of intimate partners: A meta-analysis. Journal of Research in Personality, 44, 124-127. doi:10.1016/j.jrp.2009.09.004.

Plan Nacional Sobre Drogas (2012). Encuesta sobre uso de drogas en Enseñanzas Secundarias en España (ESTUDES 1994-2012).

Substance Abuse and Mental Health Services Administration (2014). Results from the 2013 National Survey on Drug Use and Health: Summary of National Findings. Recuperado el 29 de julio de 2015, de http://www.samhsa.gov/data/sites/default/files/NSDUHresultsPDFWHTML2013/Web/NSDUHresults2013.pdf.

Rodríguez-Martos, A., Gual, A., y Llopis, J. J. (1999). La unidad de bebida estándar como registro simplificado del consumo de bebidas alcohólicas y su determinación en España. Medicina clínica, 112, 446-450.

Rose, R. J., y Dick, D. M. (2004). Gene-environment interplay in adolescent drinking behavior. Alcohol Research and Health, 28, 222-229.

Satorra, A. y Bentler, P. M. (2001). A scaled difference chi-square test statistic for moment structure analysis. Psychometrika, 66, 507-514.

Shelton, K. K., Frick, P. J. y Wootton, J. (1996). Assessment of parenting practices in families of elementary school-age children. Journal of Clinical Child Psychology, 25, 317-329.

Simons-Morton, B., y Chen, R. S. (2006). Over time relationships between early adolescent and peer substance use. Addictive Behaviors, 31, 1211-1223. doi:10.1016/j.addbeh.2005.09.006. 
Wills, T. A., y Cleary, S. D. (1999). Peer and adolescent substance use among 6th-9th graders: latent growth analyses of influence versus selection mechanisms. Health Psychology: Official Journal of the Division of Health Psychology, American Psychological Association, 18, 453-463. doi:10.1037/0278-6133.18.5.453. 